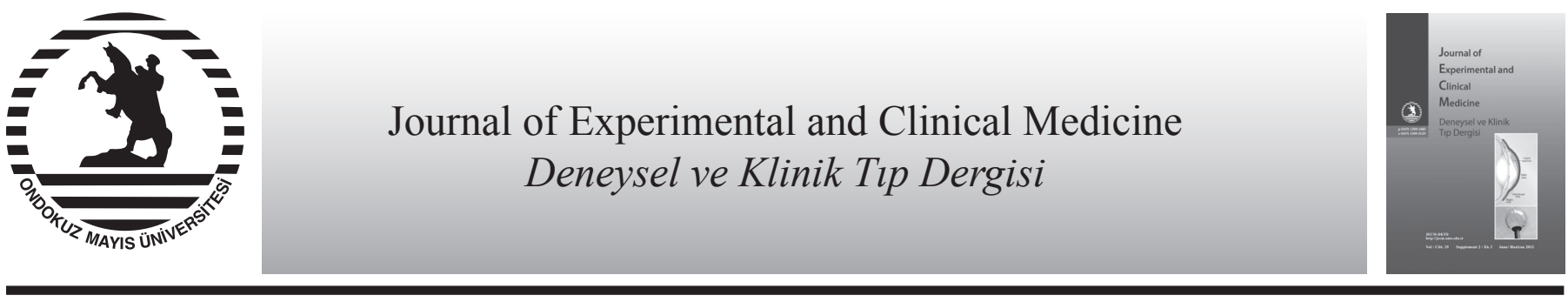

Review

doi: $10.5835 /$ jecm.omu.29.s2.002

\title{
A practical approach to evaluation of patients with diplopia
}

Nurhan Torun

Department of Ophthalmology, Harvard Medical School, Beth Israel Deaconess Medical Center, Boston, MA, USA

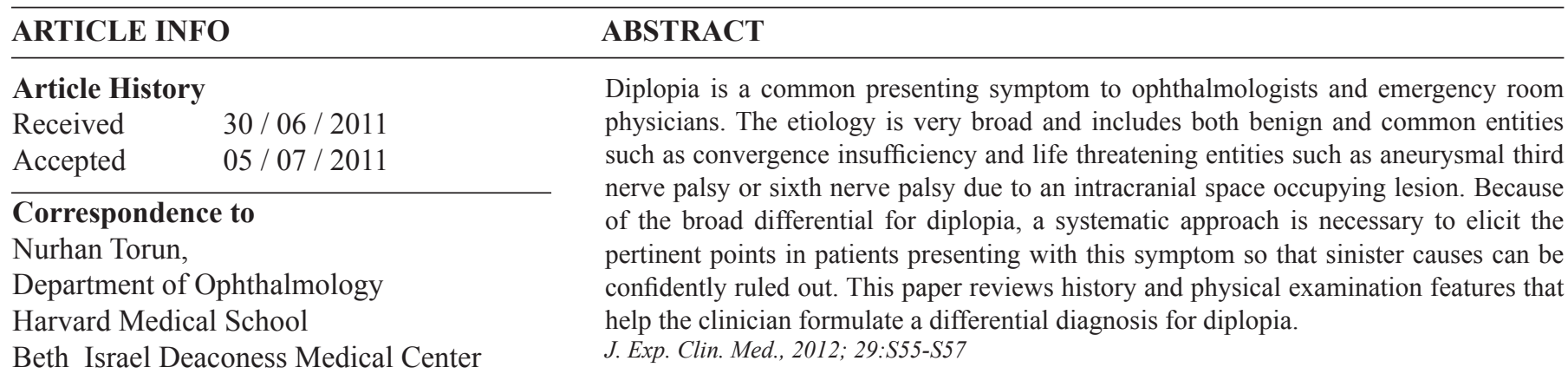

330 Brookline Avenue, Boston, MA02215,

USA

e-mail: ntorun@bidmc.harvard.edu

Keywords:

Diplopia

Etiology

Diagnosis

(C) 2012 OMU

\section{Introduction}

Diplopia is a frequently encountered symptom in eye clinics. Causes of diplopia range from benign and common entities such as a decompensated phoria to ominous entities such as aneurysmal third nerve palsy or sixth nerve palsy due to increased intracranial pressure. The presentation can be obvious and pose no diagnostic challenge; or it may be complex and require neuroophthalmic assessment. Given that the etiology of diplopia spans such a broad spectrum, it is important to have a systematic approach in evaluating patients presenting with this symptom.

\section{Table 1. Anatomical differential diagnosis for binocular diplopia}

Myopathies involving extraocular muscles ( e.g. Thyroid ophthalmopathy, chronic progressive external ophthalmoplegia and other myopathies)

Neuromuscular junction disorders (Myasthenia, botulismus)

Orbital processes (e.g. Orbital pseuodotumor, orbital cellulitis, fractures)

Cavernous sinus and superior orbital fissure processes (e.g. Carotid cavernous fistula, cavernous sinus thrombosis)

Subarachnoid space ( Isolated or combined cranial nerve palsies)

Brainstem lesions (Nuclear or fascicular ocular motor cranial neuropathies, skew deviation)

Neurodegenerative conditions (e.g. PSP, advanced Parkinson's)

Strabismic disorders (e.g. Esotropia associated with divergence insufficiency)

Others: Miller Fisher syndrome, thiamine deficiency, Whipple's disease, epiretinal membrane
Table 1 lists the anatomical differential diagnosis that we need to keep in mind when assessing patients with binocular diplopia (Torun, 2008).

\section{Important points in history}

As with most symptoms, a good history is invaluable. Sometimes history alone may point towards the diagnosis in diplopia.

\subsection{Description of diplopia}

Is diplopia monocular or binocular?

The first thing to determine is whether diplopia is monocular (persists when one eye is closed) or binocular (resolves with closure of either eye). Monocular diplopia is almost always secondary to an ocular problem such as media opacity or uncorrected refractive error and resolves with a pinhole. Monocular diplopia which does not resolve with a pinhole may very rarely be due to "central polyopia" caused by a lesion involving the visual cortex; there is usually an associated visual field defect. The focus of this paper is binocular diplopia.

\section{Is it intermittent or constant?}

Intermittent diplopia can be caused by one of the entities listed in Table 2 (Torun, 2008). The most common causes of intermittent diplopia by far are decompensating phorias and vergence problems. In patients over age 60 , it is important to think of temporal arteritis and do a review of systems to check for any red flags. 


\section{Is the separation mainly horizontal or vertical?}

This helps narrow down the group of muscles that will be considered. Most cases of pure vertical diplopia are due to fourth nerve palsy. Sixth nerve palsy causes horizontal diplopia but a small vertical misalignment may also be present and does not necessarily suggest multiple cranial nerve involvement (Wong et al., 2002). Third nerve palsies typically cause oblique separation of two images. Orbital processes can cause horizontal, vertical or oblique diplopia.

Table 2. Differential diagnosis for intermittent diplopia

Decompensating phoria

Ocular myasthenia

Spasm of accommodation

Ischemia of extraocular muscles (as in temporal arteritis)

Transient ischemia involving the vertebrobasilar system

Internuclear ophthalmoplegia

Superior oblique myokymia

Rare causes: Ocular neuromyotonia, cyclic esotropia etc.

Is it worse at distance or near?

As a general rule, horizontal diplopia that is worse at distance is generally associated with an esotropia and implies a lateral rectus problem (as in sixth nerve palsy). Conversely if diplopia is mainly reported at near, medial recti are implicated and the likely diagnosis is convergence insufficiency.

\subsection{Onset}

Diplopia is always sudden onset; however, it may start out only in certain positions of gaze and then evolve to involve primary position. It may also start intermittently and later become consistent. This type of slow progression is common for divergence insufficiency.

\subsection{Associated symptoms}

The presence of any associated symptoms can be very helpful. For example intermittent lid droopiness involving one eye or both eyes is very suspicious for myasthenia, especially if diplopia also worsens later in the day. Patients with vertebrobasilar ischemia may experience vertigo or speech difficulty along with diplopia. Most ischemic ocular motor cranial neuropathies are associated with mild ipsilateral eye pain, which generally subsides within a week. Severe pain, if present, is a red flag for temporal arteritis or subarachnoid hemorrhage. A painful third nerve palsy should make the examiner think of an aneurysmal etiology.

\subsection{Medical history}

Risk factors for ischemic ocular motor cranial neuropathy are diabetes, hypertension, and hyperlipidemia. History of thyroid problems may bring to mind Graves' disease. The most common reason patients with Parkinsonian syndromes are referred to the eye clinic is convergence insufficiency.

All patients with pure vertical diplopia should be questioned about trauma since acquired fourth nerve palsy is most commonly trauma related.

\section{Examination in diplopia}

In patients with an obvious ocular misalignment such as esotropia, exotropia or hypertropia, cover/uncover test, in conjunction with a prism bar, is used to characterize the devia- tion. It also allows quantification of the ocular misalignment. If there is no obvious misalignment, cover/uncover test and alternate cover tests can be used to look for a phoria, especially if the patient complains of intermittent diplopia.

Once misalignment is measured in primary position with the prism bar, the measurement can then be repeated in right and left gazes, up and down gazes and with right and left head tilt. In comitant deviations, the measurements do not vary much in different positions of gaze. Paralytic misalignment, on the other hand, increases with gaze towards the direction of the action of the muscle involved and vice versa; it is incomitant. Misalignment also increases when the patient fixates with the paretic eye (secondary deviation) as opposed to the uninvolved eye (primary deviation) so it is important to make note of which eye is fixating when prism measurements are documented.

The next step is to look at versions and ductions and make note of restriction observed in any position of gaze. Abduction is restricted in sixth nerve palsies. Any combination of adduction, elevation and depression may be restricted with a third nerve palsy. There is usually no restriction with fourth nerve palsies but the patient may have ipsilateral inferior oblique overaction. Extraocular myopathies such as Graves' disease frequently cause restriction in multiple directions. It is important to distinguish this from multiple ocular motor cranial nerve palsies; presence of proptosis, lid edema, lid retraction or lid lag all point towards Graves' disease, although there are cases who do not exhibit any of these signs. Any fatiguability or variability during examination should be noted and myasthenia should be considered.

Parks' three step test has been recommended as a diagnostic tool for vertical misalignments but this test only works when there is a single extraocular muscle involved; an assumption that may not hold true (Kushner, 1989). So in practice, the most useful application of Parks' three step test is a superior oblique palsy where the vertical misalignment increases with gaze towards the uninvolved eye and ipsilateral head tilt.

Skew deviation is a prenuclear vertical misalignment which is associated with brainstem and cerebellar lesions. It may be comitant or incomitant; motility is usually full and so it can be confused with a fourth nerve palsy. There is a marked decrease in the vertical misalignment when the patient lays supine, which helps differentiate skew deviation from fourth nerve palsy (Parulekar et al., 2008).

\subsection{Other findings}

It is important to examine the lid position and the pupils; both may be involved with a third nerve palsy. Unilateral or bilateral ptosis, especially if variable, should prompt workup for myasthenia. Lid edema, lid retraction or lid lag all suggest Graves' disease, as does proptosis. Sometimes a retinal lesion such as an epiretinal membrane may cause central binocular diplopia (De Pool et al., 2005). This may be hard to diagnose if the retinal findings are subtle. In patients with sixth nerve palsy, it is important to look for papilledema to determine if the patient has increased intracranial pressure. It is also important to examine the pupils carefully, since sixth nerve palsy associated with Horner syndrome signifies a cavernous sinus lesion.

In patients with third, fourth or sixth cranial nerve involvement, it is necessary to do a basic cranial nerve exami- 
nation because simultaneous multiple cranial nerve palsies require imaging and further testing.

The red flags to watch out for in patients presenting with diplopia are pupillary involvement in a third nerve palsy (aneurysm), new headache associated with intermittent diplopia (temporal arteritis) or sixth nerve palsy (intracranial mass); multiple cranial nerve involvement or presence of other neurological symptoms such as ataxia, vertigo, weakness or sensory changes (neoplasm or stroke) and variability and fatiguability (myasthenia).

\section{Conclusion}

Diplopia may be the initial presentation of a potentially life threatening disorder or it may be secondary to a harmless process. In some cases examination findings may be subtle, making it difficult to distinguish the former from the latter. Careful analysis of ocular motility examination, coupled with pattern recognition is necessary to narrow down the differential.

\section{REFERENCES}

De Pool, M.E., Campbell, J.P., Broome, S.O., Guyton, D.L., 2005. The dragged fovea-diplopia syndrome: clinical characteristics, diagnosis and treatment. Ophthalmology. 112, 1455-1462.

Kushner, B.J., 1989. Errors in the three-step test in the diagnosis of vertical strabismus. Ophthalmology. 96, 127-132.

Parulekar, M.V., Dai, S., Buncic, J.R., Wong, A.M., 2008. Head position-dependent changes in ocular torsion and vertical misalignment in skew deviation. Arch Ophthalmol. 126, 899-905.

Torun, N., 2008. Examination of the ocular motor system.In Aydin O’Dwyer P, Kansu T and Torun N, editors. Neuro-Ophthalmology Manual, Gunes Medical Publishing, Ankara. 19-28.

Wong, A.M., Tweed, D., Sharpe, J.A., 2002. Vertical misalignment in unilateral sixth nerve palsy. Ophthalmol. 109, 1315-1325. 